\title{
MIDDLE MIOCENE (BADENIAN) CONIDAE FROM LĂPUGIU DE SUS, ROMANIA: SYSTEMATICAL AND PALAEOECOLOGICAL DATA
}

\author{
CARMEN CHIRA ${ }^{1}$, ILEANA VOIA ${ }^{1}$
}

\begin{abstract}
The paper presents in synthesis the conids from Lăpugiu de Sus, especially those which are preserved in the collections of PalaeontologyStratigraphy Museum of the Babeş-Bolyai University. Conids, carnivorous gastropods who lived in shallow waters from neritic zones, in warm seas, respectivelly subtropical to tropical, with a normal salinity, have been the most diversified gastropods at Lăpugiu de Sus during the Middle Miocene (Badenian). There are also species which could tolerate slight decreasing of salinity such as Conus (Conolithus) dujardini brezinae. It was remarked that the conids from Lăpugiu de Sus lived in the conditions of shore-offshore zones where the temperature of water was about $21^{\circ} \mathrm{C}$.
\end{abstract}

KEYWORDS: Gastropods, Conidae, Middle Miocene (Badenian), systematic, palaeoecology, collections, Cluj - Napoca Museum.

\section{INTRODUCTION}

The famous fossiliferous site from Lăpugiu de Sus is located in the Mureş River passageway. The Neogene sedimentary is situated between andesitic agglomerates in the north and the Poiana Ruscă Mountains`s epicrystalline in the south.

At Lăpugiu de Sus Early Badenian deposits occur. Papp (1976) separated within these deposits the lower boundary of the Early Badenian, which can be followed in few sections cropping out on Cruşița Vinii Valley, and Fântânii Valley. The upper boundary of Early Badenian is outcropping in well-opened sections, such as those from Coşului Valley, Munteanului Valley, and Berii Valley. Most of the studied conids are from Coşului Valley.

The very rich fauna from the Early Badenian deposits from Lăpugiu de Sus was mentioned since 1845 by Bielz. Palaeontological and stratigraphical data on Lăpugiu de Sus Basin are given in Stur (1863), Hauer \& Stache (1863), Halovats (1876), Hoernes \& Auinger (1879), Koch $(1898,1900)$, Kadic (1906), Nițulescu (1930), Moisescu (1955), Orăşanu et al. (1971), Papp (1976), Petrescu et al. (1990), Chira (1994), a. o.

In 1900, the mollusks list from Lăpugiu de Sus already contained a number of 833 species specified by Koch. This number was enlarged subsequently by numerous researches, thus today there are more than thousand species.

Among mollusks, the gastropods have a higher frequency and among gastropods most of species belong to Conidae.

In a monographic study about the Neogene, Koch (1900) had already specified the presence of 45 species of Conus, the most representative genus from

I "Babeş-Bolyai" University, Department of Geology, Str. Kogălniceanu 1, 3400 Cluj-Napoca, Romania. 
Lăpugiu de Sus. Nițulescu (1930) mentioned seven species, Moisescu (1955) six species, Papp (1976) - fourteen (all these being already mentioned by Koch, 1900).

\section{SYSTEMATIC ACCOUNT}

In the Paleontology-Stratigraphy Museum from Babeş-Bolyai University (Faculty of Biology and Geology) there are eight collections, respectively: Koch, Melka, Protescu, Buda, Papp, Şuraru, Nițulescu, and Chira.

We analysed both the Conidae collections exposed in the PaleontologyStratigraphy Museum and the samples recently collected, and we noticed the existence of a number of 37 species and subspecies. The most frequent species are: Conus (Conolithus) dujardini dujardini DESHAYES and C. (Lithoconus) mercatti miocensis SACCO, 1893.

The species represented only by one specimen are:

C. (Leptoconus) tarbelianus GRATELOUP;

C. (Chelyconus) marii SACCO, 1893;

C. (Chelyconus) noe BROCC, 1814;

C. (Chelyconus) mucronatolaevis SACCO, 1893;

C. (Lithoconus) daciae HOERNES \& SACCO, AUINGER 1893;

C. (Stephanoconus) subnocturnus d'ORBIGNY;

C. (Stephanoconus) subigranosus SACCO, 1893;

C. (Dendroconus) voeslauensis HOERNES \& AUINGEER.

The maximum size belongs to $C$. (Lithoconus) mercatti subaustriaca SACCO, 1893 which has $93,63 \mathrm{~mm}$ in height $(\mathrm{H})$ and $61,24 \mathrm{~mm}$ in width $(\mathrm{L})$. The species Conus (Conolithus) dujardini dujardini DESHAYES has the smaller dimensions, $11,72 \mathrm{~mm}$ in height and $5,62 \mathrm{~mm}$ in width respectively.

The Conidae systematics from Lăpugiu de Sus (Tab. 1) was followed after Strausz (1966), and also with references to Baluk (1997). To actualise these species of Conus according to the current synonymy we used the papers of Baluk (1997) taking into consideration also the papers of Hoernes (1856), Koch (1900), Friedberg (1931 - 1934), Csepreghy-Meznerics (1956), Kojumdgieva \& Strachimirov (1960), Hall (1964), Nicorici \& Sagatovici (1973), Papp (1976) and Svagrovsky (1981).

The Conids from the eight collections of the Paleontology-Stratigraphy Museum from Cluj - Napoca are given according to the available bibliographical data, with seven subgenera: Conolithus HERRMANNSEN, 1847; Leptoconus SWAINSON, 1840; Lithoconus MOERCH, 1850; Chelyconus MOERCH, 1852; Cleobula IREDALE, 1930; Stephanoconus MOERCH, 1852; Dendroconus SWAINSON, 1840. Among these, we were able to identify 34 species, one of them having 8 subspecies. The complete number of species and subspecies is 37 .

It must be mentioned that, according to Baluk (1997), some differences occur, comparing with Strausz (1966) (Tab. 1), concerning the synonymy. 
MIDDLE MIOCENE (BADENIAN) CONIDAE FROM LĂPUGIU DE SUS, ROMANIA

Table 1.

Systematics of the analysed conids: Class GASTROPODA,

Subclass PROSOBRANCHIA, Order NEOGASTROPODA, Suprafamily CONACEA,

Family CONIDAE SWAINSON, 1840, Genus Conus LINNE, 1758

\begin{tabular}{|c|c|c|c|}
\hline SUBGENUS & $\begin{array}{l}\text { SPECIES } \\
\text { antiqus } \\
\text { ventricosus } \\
\text { raristriatus }\end{array}$ & SUBSPECIES & $\begin{array}{l}\text { AUTHOR } \\
\text { LAMARK, } 1810 \\
\text { BRONN } \\
\text { BELLARDI \& MICH., } 1840\end{array}$ \\
\hline $\begin{array}{l}\text { Conolithus } \\
\text { HERRMANNSEN, } \\
1847\end{array}$ & $\begin{array}{l}\text { antediluvianus } \\
\text { dujardini } \\
\text { dujardini }\end{array}$ & $\begin{array}{l}\text { dujardini } \\
\text { brezinae }\end{array}$ & $\begin{array}{l}\text { BRUGUIERE, } 1792 \\
\text { DESHAYES, } 1845 \\
\text { HOERNES \& AUINGER }\end{array}$ \\
\hline $\begin{array}{l}\text { Leptoconus } \\
\text { SWAINSON, } 1840\end{array}$ & $\begin{array}{l}\text { extensus } \\
\text { tarbelianus } \\
\text { brochii }\end{array}$ & & $\begin{array}{l}\text { PARTSCH, } 1856 \\
\text { GRATELOUP } \\
\text { SACCO,1893 } \\
\end{array}$ \\
\hline $\begin{array}{l}\text { Lithoconus } \\
\text { MOERCH, } 1850\end{array}$ & $\begin{array}{l}\text { subacuminatus } \\
\text { mercatti } \\
\text { mercatti } \\
\text { mercatti } \\
\text { hungaricus } \\
\text { daciae }\end{array}$ & $\begin{array}{l}\text { miocenicus } \\
\text { subaustriaca } \\
\text { canaliculato- } \\
\text { depressa }\end{array}$ & $\begin{array}{l}\text { d'ORBIGNY, } 1852 \\
\text { SACCO, } 1893 \\
\text { SACCO, } 1893 \\
\text { SACCO, } 1893 \\
\text { HOERNES \& } \\
\text { AUINGER, } 1879 \\
\text { HOERNES \& AUINGER }\end{array}$ \\
\hline $\begin{array}{l}\text { Chelyconus } \\
\text { MOERCH,1852 }\end{array}$ & $\begin{array}{l}\text { vindobonensis } \\
\text { fuscocingulatus } \\
\text { suessi } \\
\text { enzesfeldensis } \\
\text { puschi } \\
\text { ponderous } \\
\text { pelagicus } \\
\text { marii } \\
\text { noe } \\
\text { cf. noe } \\
\text { ottiliae } \\
\text { olivaeformis } \\
\text { mucronatolaevis }\end{array}$ & & $\begin{array}{l}\text { PARTSCH } \\
\text { \&HOERNES,1848 } \\
\text { HOERNES, } 1856 \\
\text { HOERNES \& } \\
\text { AUINGER,1879 } \\
\text { HOERNES \& } \\
\text { AUINGER,1879 } \\
\text { MICHELOTTI, } 1847 \\
\text { BROCCHI,1814 } \\
\text { BROCCHI } \\
\text { SACCO, } 1893 \\
\text { BROCCHI, } 1814 \\
\text { BROCCHI,1814 } \\
\text { HOERNES \& } \\
\text { AUINGER,1879 } \\
\text { HOERNES \& AUINGER } \\
\text { SACCO, 1893 }\end{array}$ \\
\hline $\begin{array}{l}\text { Cleobula } \\
\text { IREDALE,1930 }\end{array}$ & $\begin{array}{l}\text { Berghausi } \\
\text { subraristriatus } \\
\text { cf. subraristriatus }\end{array}$ & vaceki & $\begin{array}{l}\text { HOERNES \& } \\
\text { AUINGER, } 1879 \\
\text { COSTA, } 1866 \\
\text { COSTA, } 1866\end{array}$ \\
\hline $\begin{array}{l}\text { Stephanoconus } \\
\mathrm{MORCH}, 1852\end{array}$ & $\begin{array}{l}\text { subnocturnus } \\
\text { subigranosus }\end{array}$ & & $\begin{array}{l}\text { d'ORBIGNY, } 1852 \\
\text { SACCO, } 1893\end{array}$ \\
\hline $\begin{array}{l}\text { Dendroconus } \\
\text { SWAINSON, } 1840\end{array}$ & $\begin{array}{l}\text { betulinoides } \\
\text { voeslauensis } \\
\text { aff. piruloides } \\
\text { berghausi }\end{array}$ & bifasciolata & $\begin{array}{l}\text { LAMARK, } 1810 \\
\text { HOERNES \& } \\
\text { AUINGER, } 1879 \\
\text { DODERLEIN } \\
\text { SACCO, } 1893 \\
\end{array}$ \\
\hline
\end{tabular}


After Baluk (1997), the present - day synonymy is:

Conus (Conolithus) dujardini Deshayes, 1845

(= Conus antediluvianus Bruguieres

= Conus (Conolithus) dujardini brezinae Hoernes \& Auinger);

Conus (Lautoconus) posticestriatus Kojumdgieva, 1960

(= partim Conus (Chelyconus) suessi Hoernes \& Auinger);

Conus (Lithoconus) berghausi Michelotti, 1847

(= Conus (Dendroconus) daciae Hoernes \& Auinger

= Conus (Lithoconus) mercati daciae (Hoernes \& Auinger)

= Conus (Dendroconus) vaceki Hoernes \& Auinger

= Conus (Cleobula) berghausi vaceki Hoernes \& Auinger

= Conus (Dendroconus) voeslauensis Hoernes \& Auinger);

Conus (Lithoconus) betulinoides Lamarck, 1810

(=Conus (Dendroconus) betulinoides Lamarck);

Conus (Chelyconus) ponderosus Brocchi, 1814

(= Conus ponderosus Brocchi

= Conus (Chelyconus) enzesfeldensis Hoernes \& Auinger);

Conus (Chelyconus) pyrula Brocchi, 1814

(= ? Conus (Chelyconus) ottiliae Hoernes \& Auinger

= ? Conus (Chelyconus) olivaeformis Hoernes \& Auinger

= Chelyconus mucronatolaevis Sacco);

Conus (Chelyconus) rotundus Hoernes \& Auinger, 1879

( = partim Conus ventricosus Bronn);

Conus (Chelyconus) vindobonensis Partsch in Hoernes, 1856

( = partim Conus ventricosus Bronn

= Conus (Chelyconus) vindobonensis Partsch

$=$ ? Conus fuscocingulatus Bronn).

\section{PALAEOECOLOGICAL DATA}

\section{Bathymetry}

The bathymetric distribution of contemporaneous species of Conus (Hall, 1964) point out that most of the species can be found in shore zones or in neritic zones with shallow water. Hall (1964) assumed that it was possible that most of Conidae (from Piemont) belonging to "Burdigalian-Helvetian" could have lived in waters in the area between ebb line and about $10 \mathrm{~m}$ ( no more than $20 \mathrm{~m}$ ) depth.

It is estimated that the Conids from Lăpugiu de Sus lived in the neritic area with shallow water, except for Conus (Conolithus) dujardini, C. (Conolithus) antediluvianus and $C$. (Chelyconus) pushi, which might have lived in deeper marine waters.

\section{Feeding and substratum}

Hall (1964) assumed that Conus species from Miocene ages had different ways of feeding and that they lived in the same substratum as the contemporary ones. By analogy with present-day Conids, as well as with those from Piemont presented by Hall (1964), we may assume that most of Conids from Lăpugiu de Sus lived on, or buried in the sand, having various preferences regarding food, such as: polychaetes worms or enteropneusts, gastropods etc. 


\section{Sea-surface temperature}

In present-day areas of the Mediterranean Sea, where only one species of Conus: C. ventricosus GMELIN ( $=C$. mediterraneus HWASS) lives, the lowest temperature at the sea-surface in Adriatic Sea is about $10^{\circ} \mathrm{C}$, during four month per year and the mean value is between $18-20^{\circ} \mathrm{C}$, sligthly raised in the rest of the year. In the Ligurian and Tyrrhenian Seas sea-surface temperatures are slighty increased $\left(12-13^{\circ} \mathrm{C}\right)$ during five months starting with November.

If the temperature is an important factor that influences the entire existence of Conus, then the Early Badenian Sea from Lăpugiu de Sus area was warmer than the present-day Mediterranean Sea because here 45 species were specified (Koch, 1900).

Therefore, we may conclude that the marine paleoclimate was a tropical one where the temperature at sea-surface exceeded $20^{\circ} \mathrm{C}$.

\section{REFERENCES}

Baluk, W., 1997, Middle Miocene (Badenian) gastropods from Korytnica, Poland; Part III. Acta Geologica Polonica, 47, 1 - 2, p. 1 - 75, 25 pl., Warszawa.

Chira, C., 1994, Catalogue of the bivalvia collection of Lăpugiu de Sus preserved at the Palaeontology - Stratigraphy Museum in the University of Cluj. In vol. The Miocene from the Transylvanian Basin, Romania, p. 75 - 80, Cluj - Napoca.

Csepregy-Meznerics, I., 1956, Die Molluskenfauna von Szob und Letkes. A. Mag. Alt. Fold. Int. Evkonyve, XLV, 2, p. 363-477, 14 pl., Budapest.

Friedberg, W., 1931-1934, Mollusca Miocenica Polonica. Pars. I-Gasteropoda, 1-109, Krakow.

Halavats, G., 1876, Die mediterrane Fauna von Felso - Lapugy. Foldt. Kozl., VI, p. 229 - 240, Budapest.

Hall, C. A. Jr., 1964, Middle Miocene Conus (Class Gastropoda) from Piedmont, northern Italy. Bollettino della Soc. Paleont. Italiana, vol. 3, nr. 2, p. 111-171, tab. 20-28, 2 fig.

Hauer, F., Stache, G., 1863, Geologie Siebenbuergens, 637 p., Wien.

Hoernes, M., 1856, Die fossilen Mollusken des Tertiarbeckens von Wien. Abh. d. k. k. Geol. R. A. II., Bivalven IV, Wien.

Hoernes, R., Auinger, M., 1876 - 1891, Die Gasteropoden der Meeres Ablagerungen der ersten und zweiten Miocaenen Mediterran-Stufe. Abhandl. D. k. k. geol. R. A., XII, 382 p., Wien.

Kadik, O., 1906, A Maros balpartjan Tisza Dobra es Lapugy kornyeken elteruto hegyvidek geologiai viszouyai. Foldt. Kozl. Evi. Jel., p. 90 - 96, Budapest.

Koch A., 1898, Neuere Beobachtungen und Aufsamlungen in Felso-Lapugy. Foldt. Kozl., XXVIII, 209-226, Budapest.

Koch, A., 1900, Die Tertiarbildungen des Beckens der siebenbuergischen Landesteile II. Neogen. Mitth. a. d. Jahrb. d. kgl. ung. geol. Anst., 370 p. Budapest.

Kojumdgieva, E., Strachimirov, B., 1960, Les Fossiles de Bulgarie VII Tortonien. Ed. Acad. de Bulgarie, p. 1-317, 59 pl., 3 fig., Sofia.

Moisescu, G., 1955, Date noi asupra faunei de moluşte şi brachiopode tortoniene de la Lăpugiu de Sus. Rev. Univ. "Parhon", 8, 225-286, Bucureşti. 
Nicorici, E., Sagatovici, A., 1973, Studiul faunei Badenian superioare de la Minşul de Sus (Bazinul Zarand). An. Inst. Geol. Vol. XL, p. 111-194, Bucureşti.

Nițulescu, O., 1930, Contribuții la studiul geologic al regiunii Lăpugiu de Sus. Rev. Muz. Geol. Min., IV/1, 27-46, Cluj-Napoca.

Orăşanu, T., Marinescu, F., 1971, Biostratigrafia depozitelor neogene de pe rama nord - vestică a Munților Poiana Ruscă. D. S. Com. Geol., LVII!/4, p. 85 - 108, Bucureşti.

Papp, C., 1976, Biostratigrafia Neogenului din regiunea Lăpugiu de Jos - Lăpugiu de Sus-Crivina (jud. Hunedoara). D., p. 1-154, 14 fig., 43 pl., Cluj-Napoca.

Petrescu, I., Meszaros, N., Chira, C., Filipescu, S., 1990, Lower Badenian paleoclimate at Lăpugiu de Sus (Hunedoara Country), on accont of paleontological investigation. Studia Univ. Babeş-Bolyai, Geologia, XXXV, 2, p. 13-22, 5 pl., 2 fig., 1 tab., Cluj-Napoca.

Strausz, L., 1966, Die Miozaen-Mediterranean Gastropoden Ungarns. Akad. Kiado., 639p., Budapest.

Stur, D., 1863, Bericht uber die geologische Ubersichtaufnahme des sudvestlichen Sieberburgens im Sommer 1860. Jahrb. d. k. k. Geol. R. A. XIII, 33-120, Wien.

Svagrovsky, J., 1981, Lithofazielle Entwicklung und Molluskenfauna des oberen Badeniens (Miozaen M4d) in den Gebiet Bratislava-Devinska Nova Ves. Zapadne Karpaty, ser. Paleontologia 7, p., 5-204, 2. Tab., 5 texttab., LIII Taf., Bratislava.

Voia, I., 1998, Studiul sistematic şi paleoecologic al gastropodelor de la Lăpugiu de Sus. D., 70 p., 5 pls., Cluj - Napoca.

\section{PLATE I:}

1.a, b; 4.a, b Conus ventricosus BRONN - Inv. 11271, x 0.86; Inv. 1126, x 1.71

2.a, b Conus raristriatus BELLARDI \& MICHELOTTI - Inv. 11277, x 0.87

3.a, b Conus antiqus LAMARCK - Inv. 2651, x 1.73

5.a, b Conus (Conolithus) dujardini dujardini DESHAYES - Inv. 23212, x 2.13

6.a, b Conus (Conolithus) antediluvianus BRUGUIERE - Inv. 11276, x 1.51

\section{PLATE II:}

1.a, b Conus (Leptoconus) extensus PARTSCH - Inv. 1842, x 0.69

2.a, b Conus (Lithoconus) mercatti caniculatodepresa SACCO - Inv. 12479, x 0.87

3.a, b Conus (Lithoconus) mercatti miocenicus SACCO - Inv. 23214, x 1

4.a, b Conus (Lithoconus) mercatti subaustriaca SACCO - Inv. 23211, x 0.90

5.a, b Conus (Chelyconus) vindobonensis PARTSCH - Inv. 2613, x 1.49

\section{PLATE III:}

1.a, b Conus (Chelyconus) ponderosus BROCCHI - Inv. 2509 B, x 0.86

2.a, b Conus (Chelyconus) noe BROCCHI - Inv. 12216, x 0.96

3.a, b Conus (Chelyconus) enzesfeldensis HOERNES \& AUINGER - Inv. 18961, x 1

\section{PLATE IV:}

1.a, b Conus (Chelyconus) puschi MICHELOTTI - Inv. $1843 \mathrm{~A}, \times 1.07$

2.a, b Conus (Chelyconus) marii SACCO - Inv. 11280, x 0.85

3.a, b Conus (Dendroconus) betulinoides LAMARCK - Inv. 11273, x 0.87

4.a, b Conus (Dendroconus) voeslauensis HOERNES \& AUINGER - Inv. 18953, x 1.52

5.a, b Conus (Cleobula) berghausi vaceki HOERNES \& AUINGER - Inv. 2593, x 1.95. 
MIDDLE MIOCENE (BADENIAN) CONIDAE FROM LĂPUGIU DE SUS, ROMANIA
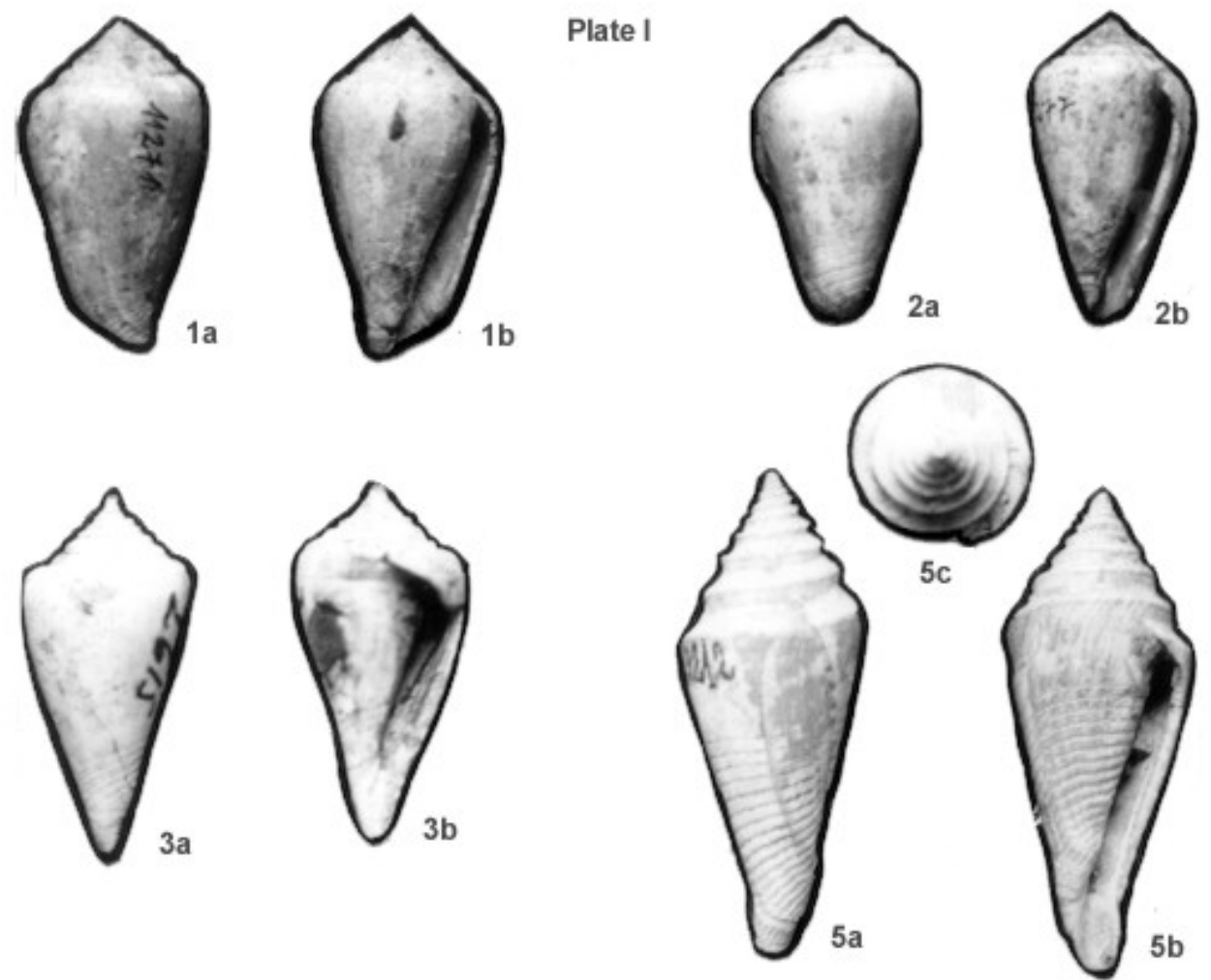

$5 c$
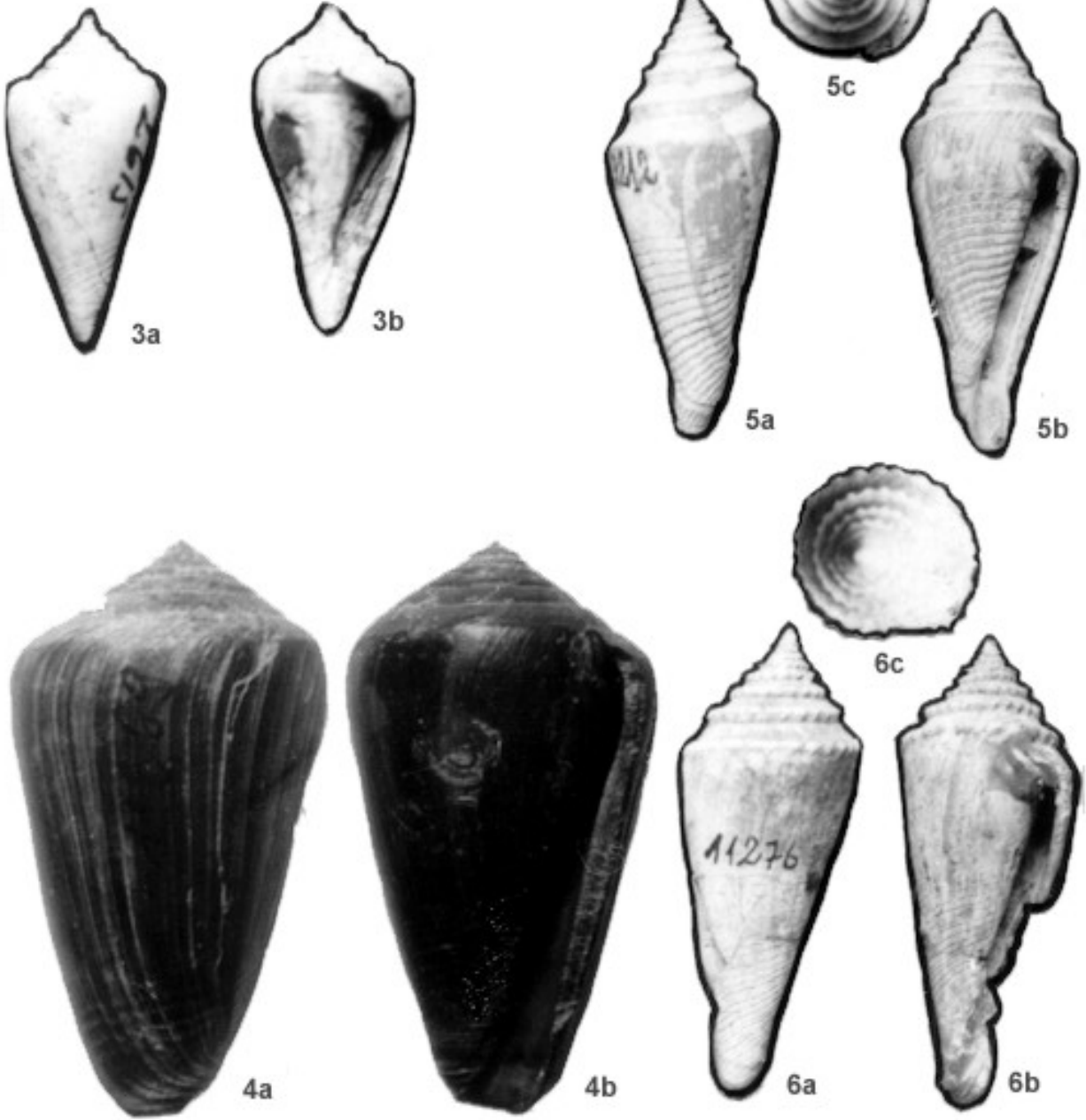
CARMEN CHIRA, ILEANA VOIA
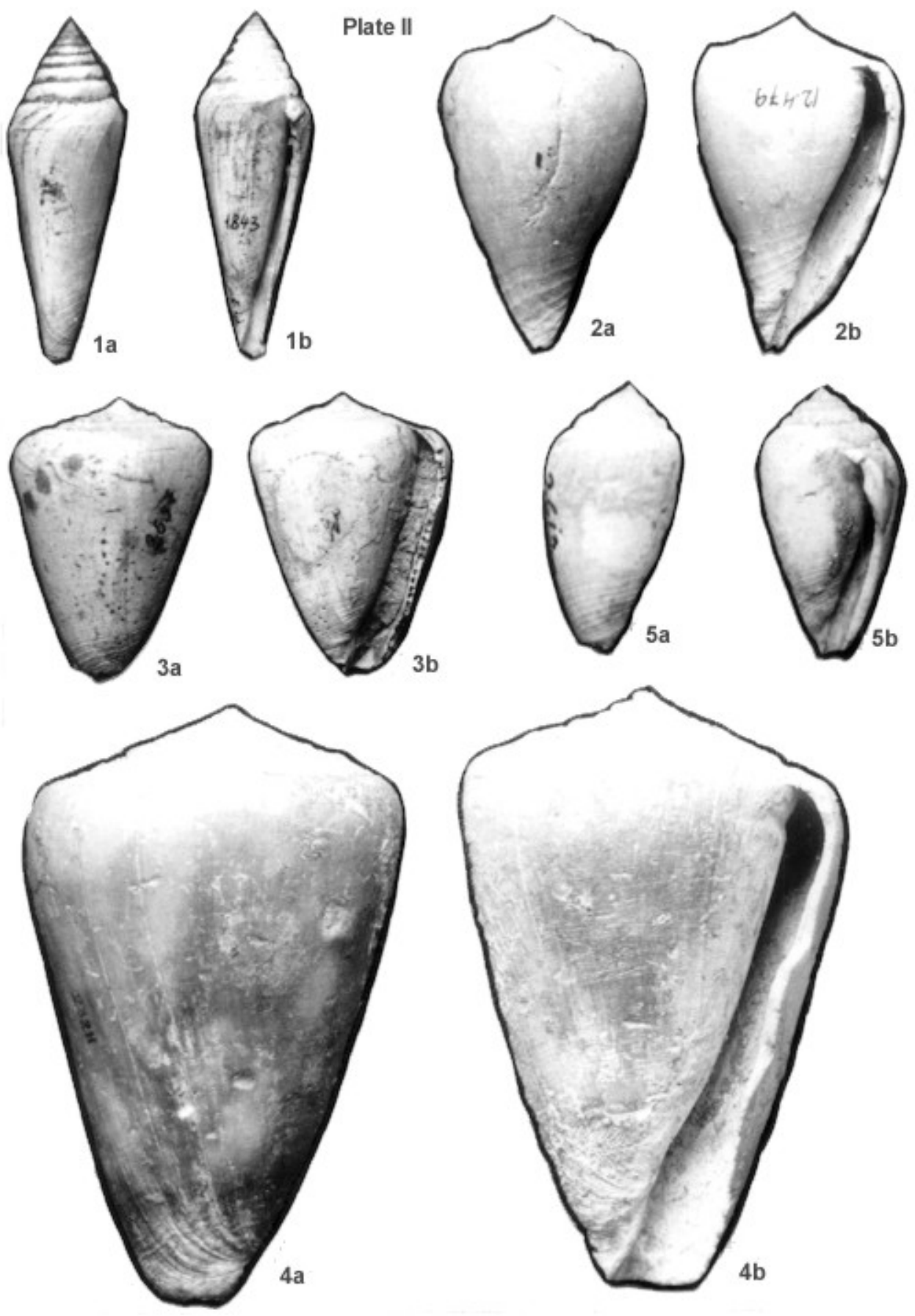
MIDDLE MIOCENE (BADENIAN) CONIDAE FROM LĂPUGIU DE SUS, ROMANIA

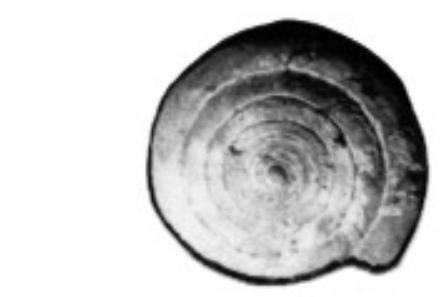

Plate III
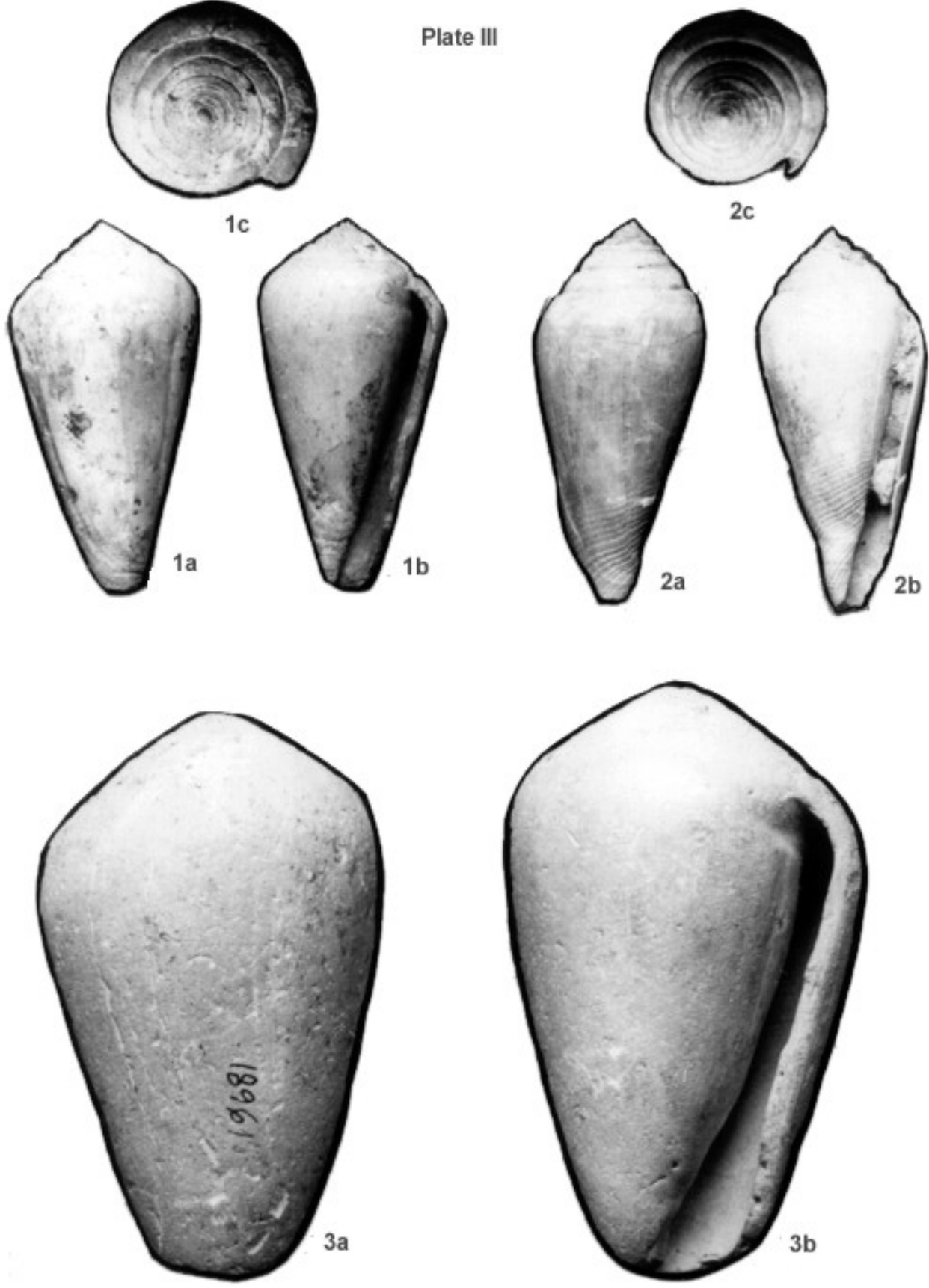
CARMEN CHIRA, ILEANA VOIA
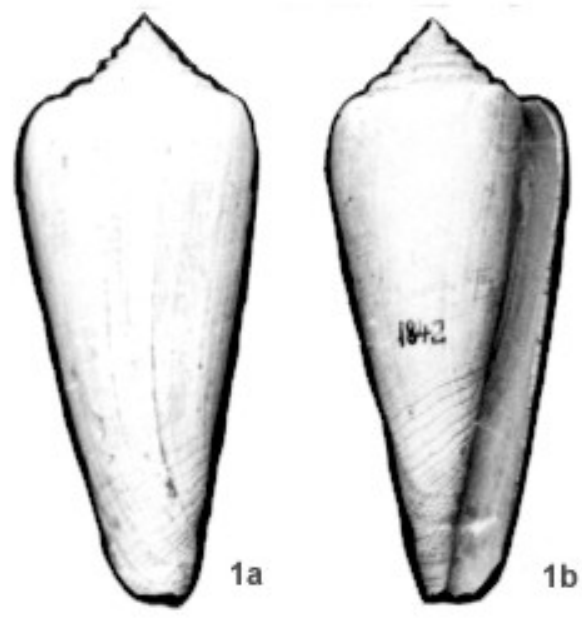

Plate IV
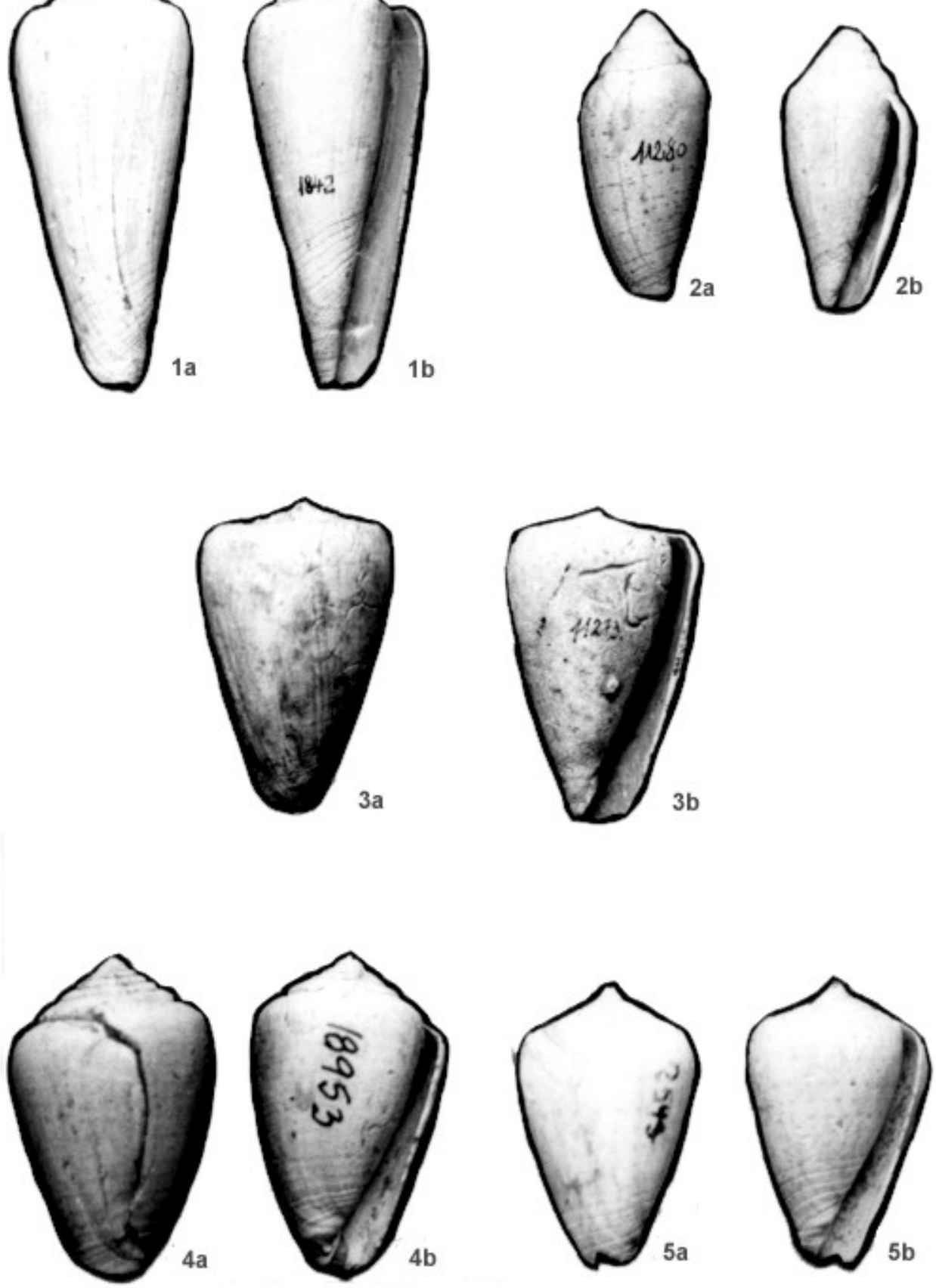